\title{
OBSERVATION OF ANOMALOUS RESISTIVITY \\ CAUSED BY ION ACOUSTIC TURBULENCE
}

Y. Kawai and M. Guyot

Centre de Recherches en Physique des Plasmas

Ecole Polytechnique Fédérale de Lausanne 
OBSERVATION OF ANOMALOUS RESISTIVITY

CAUSED BY ION ACOUSTIC TURBULENCE

Y. KAWAI and M. GUYOT

Abstract

The dc resistivity caused by ion acoustic turbulence in plasmas is studied experimentally. Below approximately $0.2 \mathrm{~V} / \mathrm{cm}$, the resistivity increases as $\mathrm{E}$. Above $0.6 \mathrm{~V} / \mathrm{cm}$, the resistivity varies as $\mathrm{E}^{\frac{1}{2}}$. These experimental results can be explained by using the Horton et al.'s theoretical mode1, in which de nonlinear ion Landau damping dominates turbulence spectrum. 
Observation of anomalous electrical resistivity caused by ion acoustic turbulence in plasmas has been reported by many authors \{1-6\}. Collective electric fields associated with the ion acoustic instability $\{7\}$ provide an effective high collision rate for electrons, which, experimentally, appears as anomalously high electrical resistivity. Therefore, measurements of turbulence spectrum are very important for explaining the anomalous resistivity quantitatively. However, the relationship between the anomalous resistivity and the turbulence spectrum has not been investigated experimentally in detail so far. We wish to report here that the anomalous resistivity caused by the current-driven ion acoustic instability was observed in a large diameter plasma and the dependence of the resistivity on electric fields can be explained by using the theoretical results which have been derived by Horton et al. \{8-9\}.

The experiments were performed using the plasma box $\{10\}$, whose diameter and length were $70 \mathrm{~cm}$ and $35 \mathrm{~cm}$, respectively. The plasma has been produced by discharge between the tungsten filaments and the chamber wall with permanent magnets. The density and temperature of the electron were measured by the Langmuir probe and the electron energy analyzer, and were found to be homogeneous accross the box. The electron density $\mathrm{n}_{\mathrm{e}}$ was varied from $10^{9}$ to $5 \times 10^{10} \mathrm{~cm}^{-3}$ by changing the emission currents of the filaments. The electron temperature $T_{e}$ was in the range of $1-3 \mathrm{eV}$. The pressure of the Argon gas was varied from $5 \times 10^{-5}$ to $5 \times 10^{-4}$ Torr. The temperature ratio $\mathrm{T}_{\mathrm{e}} / \mathrm{T}_{\mathrm{i}}$, which was obtained by taking the dispersion relation of the test ion acoustic wave, was found from 10 to 15 .

The current-driven ion acoustic instability was excited by drifting the electrons in the plasma. The drift motion of the electron was caused $\{11-12\}$ by the dc electric fields between two grids, $G_{1}$ and $G_{2}$ 
(5 $\mathrm{cm}$ in diameter), which were relatively coarse in order to avoid the disturbance to the plasma (size of mesh $\gg$ Debye length). The potential $\mathrm{V}_{\mathrm{g}}(0-250 \mathrm{~V})$ was applied to the grid $G_{l}$. Although $\mathrm{V}_{\mathrm{g}}$ was relatively large, electron beams, which may be produced by the grids, were not observed. The unstable waves were detected by the grids and the probes.

The electric field $\mathrm{E}$ was observed by measuring the floating potential on the thin two probes spaced $1 \mathrm{~cm}$ apart, which were placed at the center between two grids. A directional rotating probe $\{5\}$, located in the plasma between the two grids, was used to measure the electron drift velocity $v_{d}$. Thus, we can determine the electrical resistivity $\rho$ experimentally by using $\rho=E / e n v d$.

When the grid potential $\mathrm{V}_{\mathrm{g}}$ exceeds a critical value ( $\left.10 \mathrm{~V}\right)$, the ion acoustic waves have been observed in the plasma between the two grids $G_{1}$ and $G_{2}$. Increasing $V_{g}, v_{d}$ increased up to $0.15 v_{e}\left(=\sqrt{T_{e} / m}\right)$ and the fluctuation level of the waves did up to $15 \%$. At the same time, the peak frequency of the power spectrum shifted towards the low frequencies, which is due to some nonlinear process $\{8-9,13\}$. We show a typical example of the final power spectrum in Fig. 1, where the current fluctuation was about $6 \%$ and the distance, $\mathrm{L}$, between the two grids was $7 \mathrm{~cm}$. Figure 1 shows that the power spectrum is proportional to $\omega^{-1}$ around the peak frequency and decreases more rapidly than $\omega^{-1}$ in the high frequencies.

For the case where the nonlinear ion Landau damping dominates the stationary wave spectrum, Horton et al. \{8-9\} have obtained a modified Kadomtsev spectrum $\{13\}$ by a renormalized theory, including a modification of an electron distribution due to the feedback effect of the turbulent waves. In order to compare our experimental results with their theory, we plotted the Horton et al.'s spectrum $\{8-9\}$ as the dashed line in Fig.1 
indicating a good agreement. In this case, the theoretical curve was chosen so as to fit the experimental results. Thus, one can say that in the present experiment the nonlinear ion Landau damping plays an important role in the establishment of the turbulence spectrum.

We measured the mobility in the plasma with the ion acoustic turbulence described above. An example of our mobility measurements for the different plasma density as a function of the electric fields is shown in Fig. 2, where $\mathrm{T}_{\mathrm{e}}=1 \mathrm{eV}$ and the pressure was $2 \times 10^{-4}$ Torr. As seen from Fig. 2, the mobility $\mu$ is found to have the following power laws : (1) $\mu=$ const. for $\mathrm{E} \leqslant 0.06 \mathrm{~V} / \mathrm{cm}$, (2) $\mu \alpha \mathrm{E}^{-1}$ for $0.06 \mathrm{~V} / \mathrm{cm}$ $\leqslant E \leqslant 0.2 \mathrm{~V} / \mathrm{cm}$, and (3) $\mu \alpha \mathrm{E}^{-\frac{1}{2}}$ for $\mathrm{E} \geqslant 0.6 \mathrm{~V} / \mathrm{cm}$.

The highest mobility in Fig. 2 is already a factor of 30 lower than the value due to classical collisions $\nu_{c}$, as observed elsewhere $\{5\}$. In this case, we took the length of the plasma box as a mean free path since the mean free path for electron-neutral collisions was much larger than the size of the plasma.

The critical electric field, above which the mobility begins to decrease, is about $0.06 \mathrm{~V} / \mathrm{cm}$. as seen in Fig. 2. On the other hand, the Dreicer field $E_{D r} \sim 0.03 \mathrm{~V} / \mathrm{cm}$, which is about equal to the observed critical field.

In order to interprete the experiment quantitatively, we calculated the mobility numerically for different electron drift velocity by using the effective collision frequency derived by Horton et al. According to them, the effective collision frequency $\nu_{\text {eff }}$ is given as follows $\{8\}$ :

$$
\nu_{\text {eff }}=\frac{15 \pi}{64}\left(\frac{\pi}{2}\right)^{\frac{1}{2}} \frac{T_{e}}{T_{i}} \frac{v_{d}}{V_{e}} \omega_{p e} \int_{x_{\min }}^{l} d x\left[\delta \ln (1 / x)-2\left(\pi \nu_{\text {eff }} / \omega_{p e}\right)^{\frac{1}{2}}\left(x^{-\frac{1}{2}}-1\right)\right]
$$


where $\mathrm{x}_{\min }$ is determined by

$$
\delta \ln \left(x_{\min }{ }^{-1}\right)-2\left(\pi \nu_{e f f} / \omega_{p e}\right)^{\frac{1}{2}}\left(x_{\min }{ }^{-\frac{1}{2}}-1\right)=0
$$

In the above equations, $\delta=1+\gamma^{i} / \gamma_{L}^{e}$, where $\gamma^{i}$ and $\gamma_{L}^{e}$ are the damping rate for the ion and the linear growth rate for the electron, respectively. By solving the above coupled equation, we obtain $\nu_{\text {eff }}$ in terms of $\mathrm{v}_{\mathrm{d}} / \mathrm{v}_{\mathrm{e}}$, that is, $\mathrm{E}$. The result for $\mathrm{T}_{\mathrm{e}} / \mathrm{T}_{\mathrm{i}}=15$ and $\mathrm{n}_{\mathrm{e}}=2 \times 10^{10} \mathrm{~cm}^{-3}$ is shown as the dashed line in Fig. 2. In estimating $\delta$, we neglected the contribution of the high energy tail of the ions, which has been observed in most cases of the turbulent heating experiments $\{14\}$. As seen from Fig. 2, the experimental results agree with the theoretical ones .

We discuss the theoretical results. The theoretical curve in Fig. 2 demonstrates that (1) $\mu \alpha \mathrm{E}^{-1}$ for $E \leqslant 0.1 \mathrm{~V} / \mathrm{cm}$ and (2) $\mu \alpha \mathrm{E}^{-\frac{1}{2}}$ for $\mathrm{E} \approx 0.6 \mathrm{~V} / \mathrm{cm}$. In other words, the electrical resistivity $\rho$ varies as $E$ in low electric fields and it does as $E^{\frac{1}{2}}$ in high electric fields. Sagdeev and Galeev \{15\} have derived the relation $\rho \alpha E^{\frac{1}{2}}$ from the Kadomtsev spectrum, which can be understood physically as follows : For large electron drift velocity the wave energy transfers from the high frequencies to the low frequencies by the nonlinear ion Landau damping. As a result, the wave energy concentrates arount the peak frequency of the turbulence spectrum, which becomes essentially the Kadomtsev spectrum, as seen from Fig. 1. On the other hand, the effective collision frequency is proportional to the wave energy. Therefore, for large electron drift velocity, the resistivity has almost the same relation as that calculated from the Kadomtsev spectrum. Thus, we can conclude from these discussions that the anomalous resistivity observed here has been caused by the ion acoustic turbulence, in which the nonlinear ion Landau damping is the dominant process.

In summary, the ion acoustic turbulence was excited by the current-driven ion acoustic instability in a large diameter plasma. The turbulence spectrum agrees with that predicted by Horton et al. We obtained the 
anomalous resistivity of mobility caused by such turbulence by measuring the electron drift velocity and the electric field independently. To interprete the experimental results, the effective collision frequency was calculated by using the Horton et al.'s theory. We found that in the presence of the ion acoustic turbulence, in which the nonlinear ion Landau damping dominates, the resistivity increases as $E$ in low electric fields and does as $E^{\frac{1}{2}}$ in high electric fields.

We would like to thank Professor E.S. Weibel and Dr. Ch. Hollenstein for fruitful discussions. This work was supported by the Swiss National Science Foundation. 
REFERENCES

\{1\} S.D. Fanchenko, B.A. Demidov, N.I. Elagin, D.D. Ryutov, Sov. Phys. JETP 19, 337 (1964)

\{2\} B.A. Demidov, N.I. Elagin, D.D. Ryutov, S.S. Fanchenko Sov. Phys. JETP 21, 302 (1965)

\{3\} S.M. Hamberger, M. Friedman, Phys.Rev.Lett. 21, 674 (1968);

S.M. Hamberger, J. Jancarik, Phys.Fluids 15, 825 (1968)

\{4\} D. Dimok, E. Mazucato, Phys.Rev.Lett. 20, 713 (1968)

\{5\} A. Hirose, I. Alexeff, W.D. Jones, S.T. Kush, K.E. Lonngren, Phys.Rev.Lett. 25, 1563 (1970)

\{6\} N.I. Elagin, S.D. Fanchenko, Sov.Phys.JETP 40, 674 (1975)

\{7\} E.A. Jackson, Phys.Fluids $\underline{3}, 786$ (1960)

\{8\} Duk-In Choi, W. Horton Jr., Phys.Fluids 17, 2048 (1974)

\{9\} W. Horton Jr., Duk-In Choi, R.A. Kock, Phys.Rev. 14A, 424 (1976)

$\{10\}$ Y. Kawai, Ch. Hollenstein, Phys.Lett. 59A, 353 (1976)

$\{11\}$ H. Tanaca, A. Hirose, M. Koganei, Phys.Rev. 161, 94 (1967)

\{12\} N.Th Karatzas, A.J. Anastassiadio, K. Padopoulos, Phys.Rev.Lett. 35, 33 (1975)

\{13\} B.B. Kadomtsev, Plasma Turbulence (Academic Press, New York, 1965) chapter IV 
\{14\} R.D. Bentson, K.W. Gentle, J. Jancarik, S.S. Medley, P. Nielson P. Phillips, Phys.Fluids $\underline{18}, 710$ (1975)

\{15\} R.Z. Sagdeev, A.A. Galeev, Nonlinear Plasma Theory, edited by T.M. O'Neil and D.L. Book (Benjamin, New York, 1969) p. 103 


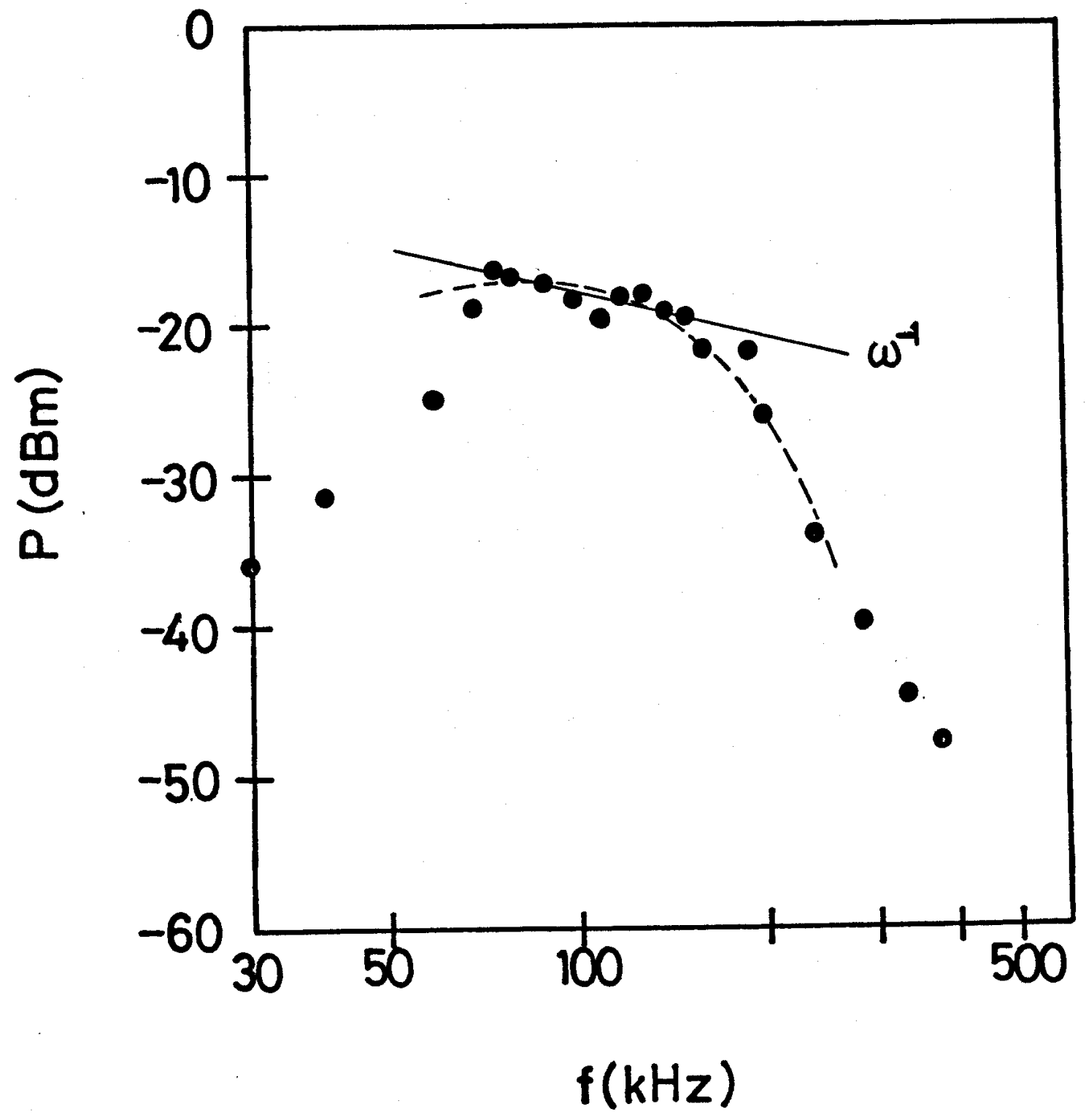

Fig. 1 


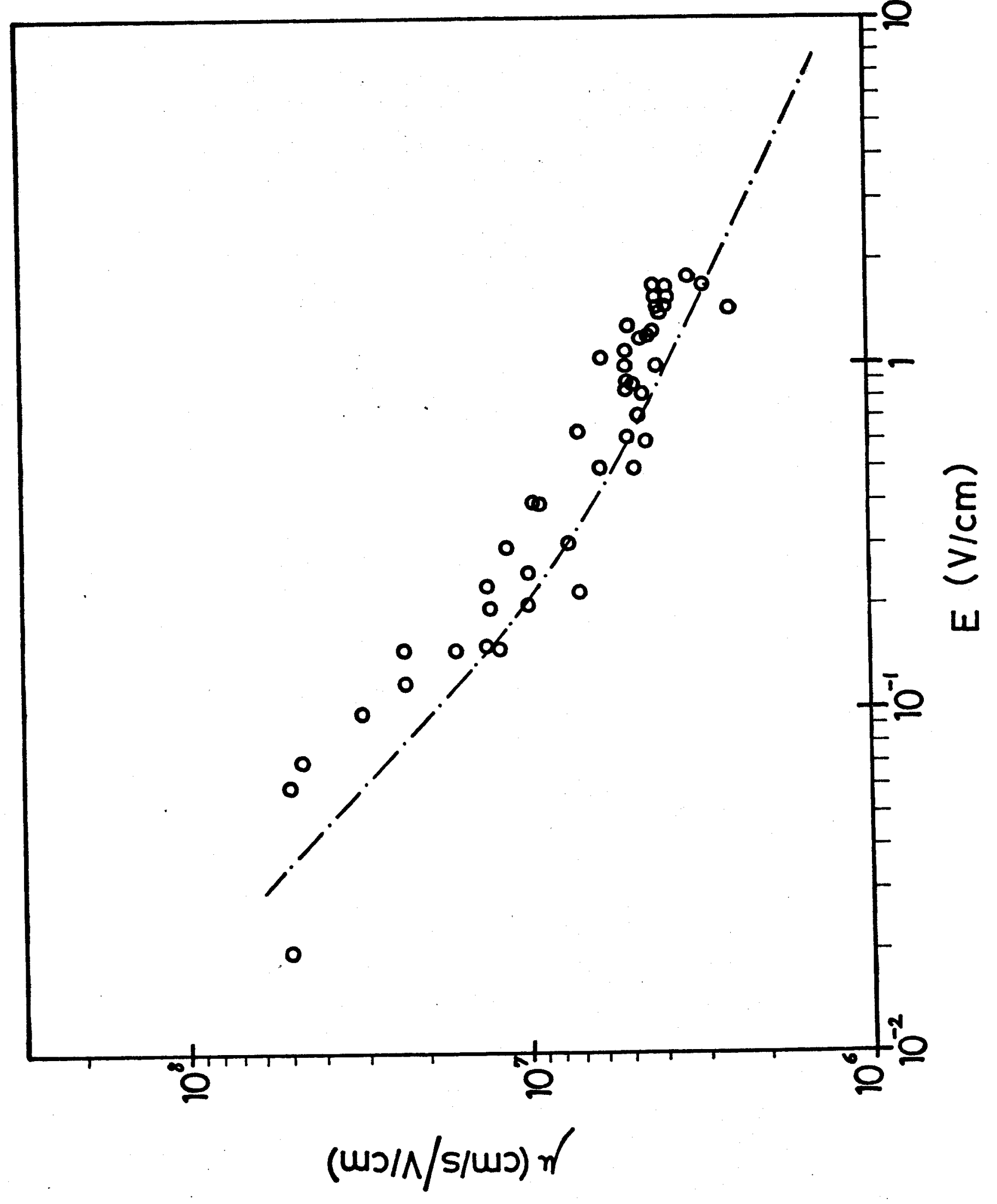

Fig. 2 